\title{
Are there any effects of Sevoflurane and Desflurane anaesthesia on blood glucose levels in acute hyperglycemic diabetic rats?
}

\author{
Dikmen $\mathrm{B}^{1}$, Arpaci $\mathrm{AH}^{2}$, Kalayci $\mathrm{D}^{3}$, Gunes $\mathrm{I}^{4}$, Beskardes $\mathrm{E}^{1}$, Kurtipek $\mathrm{O}^{1}$, Arslan $\mathrm{M}^{1}$ \\ Department of Anaesthesiology and Reanimation, Gazi University Medical Faculty, Ankara, Turkey. \\ mustarslan@gmail.com
}

\section{ABSTRACT}

AIM: The aim of this study was to investigate blood glucose level of desflurane and sevoflurane on blood glucose in diabetic rats undergoing acute hyperglycemia.

MATERIALS AND METHODS: In this study, 30 male Wistar albino rats were included. Diabetes was induced by a single IP injection of streptozotocin. After the effects of chronic diabetes encountered, diabetic rats were randomly assigned into diabetic control (group DC), diabetic hyperglycemia group (group DH), diabetic hyperglycemia group with desflurane (group DH-D), and diabetic hyperglycemia group with sevoflurane (group $\mathrm{DH}$ S) groups. The normoglycemic groups received an IP injection of the same amount of saline. Hyperglycemic diabetic rats were anaesthetized by desflurane $6 \%$ or sevoflurane $2 \%$ at a dose, by which minimal alveolar concentration (MAC) for rats would be one. The drugs were given for 4 hours within $100 \%$ oxygen at a rate of $6 \mathrm{~L}$. $\mathrm{min}^{-1}$. One hour after cessation of inhalation anesthesia, blood glucose levels were determined at 1st, 4th and 24th hours. 24 hours after the anaesthesia.

RESULTS: Serum glucose was detected to be significantly lower in Group C, when compared to Groups DC, $\mathrm{DH}, \mathrm{DH}-\mathrm{D}$ and DH-S $(\mathrm{p}=0.002, \mathrm{p}=0.001, \mathrm{p}=0.002, \mathrm{p}=0.003$, respectively).

Blood glucose levels in the diabetic groups were similar at the end of 6 weeks period, after hyperglycemia and anaesthesia induction.

CONCLUSION: We found out that sevoflurane and desflurane administrations in hyperglycemic rats were both related with insignificant blood glucose level increase at early post anaesthesia period and at post anaesthesia 24th hours. We still think that patients undergoing anaesthesia protocols with acute hyperglycemia need relatively longer follow up periods (Tab. 1, Ref. 28). Text in PDF www.elis.sk.

KEY WORDS: diabetes mellitus, acute hyperglycemia, sevoflurane, desflurane, blood glucose level, rat.

\section{Introduction}

In the recent two or three decades, the prevalence of diabetes mellitus (DM) has rapidly increased throughout the world, the estimation being that it will increase by $200 \%$ in the next few decades (1-5). As a result, physicians will be faced with an increasing population of diabetic patients undergoing anesthesia and surgery. These patients are carrying high risks for serious cardiovascular complications eventually leading to significant increases in mortality and length of stay rates in hospital (1-3).

Hyperglycemic state of body is generating serious complications, which include impaired wound healing and immune system

${ }^{1}$ Department of Anaesthesiology and Reanimation, Gazi University Medical Faculty, Ankara, Turkey, ${ }^{2}$ Department of Oral and Maxillofacial Surgery, Ankara University Faculty of Dentistry (Anaesthesiology Reanimation specialist) Besevler, Ankara, Turkey, ${ }^{3}$ Department of Anaesthesiology and Reanimation, Abdurrahman Yurtaslan Oncology Training and Research Hospital, Ankara, Turkey, and ${ }^{4}$ Department of Anaesthesiology and Reanimation, Erciyes University Medical Faculty, Kayseri, Turkey

Address for correspondenece: M. Arslan, Dr, Gazi University Medical Faculty, Department of Anaesthesiology and Reanimation, 06510 Ankara, Turkey.

Phone: +90.312 .2026739$ functions, dehydration and proteolysis at cellular and tissue level. Beside these, hyperosmolar non-ketotic comma is a well-known effect of dehydration due to high serum glucose levels. Poor wound healing in patients with diabetes is a consequence of several factors related to hyperglycemia including impaired blood flow, fibroblast activity and vitamin $\mathrm{C}$ uptake, which plays an important role in collagen synthesis (6).

Diabetes mellitus is the leading, but not the only cause of hyperglycemia in vivo. Several others include surgery and general anesthesia leads to a prompt neuroendocrine stress response identified by elevated levels of stress hormones such as: epinephrine, glucagon, cortisol, growth hormone with interleukin-6, tumour necrosis factor-alpha bursts. Activation of stress response results in increased insulin resistance in certain tissues with higher insulin levels, elevated lipolysis and protein degradation, impaired peripheral glucose utilization that results in hyperglycemia and ketosis in some states (7-10).

Different anaesthesia protocols (epidural anaesthesia is related with lower metabolic disarrangements than that general anesthesia) and surgery types (renal and liver transplantation surgery or cardiovascular bypass protocols are commonly associated with hyperglycemia and insulin resistance) lead to different levels of counter regulatory hormone release. Also, limitation or cessation 


\section{1-354}

of the caloric intake at postoperative period can result in impaired glycemic balance (10).

In vitro, volatile anaesthetics such as halothane, enflurane and isoflurane inhibit insulin responses to glucose in a reversible and dose-dependent manner (11-15). The clinical study by Diltoer and Camu showed that glucose tolerance was impaired by isoflurane. In the experimental study (14), halogenated anaesthetic agents, such as halothane or sevoflurane, produced higher negative inotropic effects in diabetic patients compared to normal myocardium, possibly because diabetes exacerbates anaesthetic-induced alterations in troponin-tropomyosin complex activity.

At present, it is uncertain, which anaesthetic agents facilitate adequate glucose control and hemodynamic stability during the perioperative period.

The aim of this study was to investigate blood glucose level of desflurane and sevoflurane on blood glucose in diabetic rats undergoing acute hyperglycemia.

\section{Material and methods}

\section{Animals and experimental protocol}

The study was performed upon the approval of Gazi University Experimental Animals Ethics Committee in Gazi University Experimental and Clinical Research Centre (GUDAM). All the procedures were performed according to the accepted standards of the Guide for the Care and Use of Laboratory Animals.

In this study, 30 male Wistar albino rats weighing between 200 and $250 \mathrm{~g}$, raised under the same environmental conditions were used. The rats were kept under $20-21^{\circ} \mathrm{C}$ at cycles of 12-hour daylight and 12-hour darkness and had free access to food until 2 hours before the anesthesia procedure.

The animals were randomly separated into five groups, each containing 6 rats: diabetic control (group DC), diabetic hyperglycemia group (group DH), diabetic hyperglycemia group with desflurane (group DH-D), and diabetic hyperglycemia group with sevoflurane (group DH-S). Another 6 rats without diabetes were assigned as the control group (group C).

STZ (Sigma Chemicals, St. Louis, MO, USA) were prepared by dissolving in saline solution $(0.9 \% \mathrm{NaCl})$. STZ was freshly prepared just before the treatment at a dose of $55 \mathrm{mg} \cdot \mathrm{kg}^{-1}$ body weight. Blood glucose levels of diabetic rats were checked by glucometer $(\mathrm{mg} / \mathrm{dl}) 3$ days after administration of STZ. Rats were classified as diabetic if their fasting blood glucose (FBG) levels exceeded $250 \mathrm{mg} \cdot \mathrm{dl}^{-1}$, and only animals with FBGs of $>250$ $\mathrm{mg} . \mathrm{dl}^{-1}$ were included in the diabetic groups (diabetes only, dia- betes hyperglycemia, diabetes hyperglycemia plus sevoflurane and diabetes hyperglycemia plus desflurane). The rats were kept alive 6 weeks after streptozotocin injection to allow the development of chronic diabetes before they were exposed to sevoflurane and desflurane.

\section{Hyperglycemia}

Hyperglycemia was induced with an intraperitoneal injection of 2.5 g. $\mathrm{kg}^{-1}$ of glucose. The normoglycemic groups received an IP injection of the same amount of saline.

Before the study was started, anaesthetic gas vaporisers were calibrated. Anaesthetic gases were set at a minimum alveolar concentration (MAC) of 1 and desflurane $6 \%$ and sevoflurane 2 $\%$ were administered. The anaesthesia procedure was conducted with the rats in a transparent plastic container of $40 \times 40 \times 70 \mathrm{~cm}$ in size. The container, which allowed for observations of the rats, was connected to a half open anaesthesia machine with static hoses. The anaesthetic gases were released into the container in $100 \% \mathrm{O}_{2}$.

The rats were divided into five groups $(n=6)$. The control, the DC and the DH groups were not subjected to any application. Desflurane (Suprane, Eczacıbaşı, İstanbul, Türkiye) was administered at $6 \%$ inspiratory concentration, 6 L.min- 1 in $100 \% \mathrm{O}_{2}$ for 2 hours, and sevoflurane (Sevorane, Abbot, Istanbul, Turkey) was administered at $2 \%$ inspiratory concentration, 6 L.min-1 in $100 \%$ $\mathrm{O}_{2}$ for 4 hours. One hour after cessation of inhalation anaesthesia, blood glucose levels were determined at 1st, 4th and 24th hours.

After anaesthesia procedure, all rats were given ketamine 100 $\mathrm{mg} \cdot \mathrm{kg}^{-1}$ intraperitoneally.

\section{Statistical analysis}

Statistical Package for the Social Sciences (SPSS, Chicago, IL, USA) 20.0 program was used for statistical analysis. The importance of the difference of the mean blood glucose levels were assessed by using the Kruskal-Wallis test. Bonferroni adjusted Mann-Whitney U test was used after significant Kruskal-Wallis to determine, which group differs from the other. Results were expressed as the mean \pm standard deviation (mean $\pm \mathrm{SD}$ ). Statistical significance was set at a $\mathrm{p}<0.05$.

\section{Results}

Blood glucose measurements were $102.50 \pm 5.24,282.25 \pm$ $32.53,316.25 \pm 54.94,298.33 \pm 30.18,276.25 \pm 263.90 \mathrm{mg} / \mathrm{dL}$ for Group C, DC, DH, DH-D and DH-S, respectively (Tab. 1).

Tab. 1. Blood glucose levels in rats (mean \pm SD).

\begin{tabular}{|c|c|c|c|c|c|c|}
\hline & $\begin{array}{c}\text { Group C } \\
(\mathrm{n}=6)\end{array}$ & $\begin{array}{c}\text { Group DC } \\
(\mathrm{n}=6)\end{array}$ & $\begin{array}{c}\text { Group DH } \\
(\mathrm{n}=6)\end{array}$ & $\begin{array}{c}\text { Group DH-D } \\
(\mathrm{n}=6)\end{array}$ & $\begin{array}{c}\text { Group DH-S } \\
(\mathrm{n}=6)\end{array}$ & $\mathrm{p}$ \\
\hline 72th hour & $102.50 \pm 5.24$ & $282.25 \pm 32.53 *$ & $316.25 \pm 54.94 *$ & $298.33 \pm 30.18 *$ & $276.25 \pm 263.90 *$ & 0.003 \\
\hline 1st hour after hyperglycemia & - & $283.00 \pm 32.25$ & $367.00 \pm 51.11$ & $332.75 \pm 28.11$ & $362.50 \pm 58.31$ & 0.537 \\
\hline 1 st hour at post-anesthesia & - & - & $328.50 \pm 34.58$ & $381.20 \pm 22.37$ & $424.00 \pm 69.19$ & 0.364 \\
\hline 4th hour at post-anesthesia & - & - & $328.00 \pm 29.08$ & $361.00 \pm 39.37$ & $371.50 \pm 45.43$ & 0.716 \\
\hline 24 th hour at post-anesthesia & - & - & $330.50 \pm 23.74$ & $365.60 \pm 29.14$ & $354.50 \pm 42.39$ & 0.741 \\
\hline
\end{tabular}


Serum glucose was detected to be significantly lower in Group C, when compared to Groups DC, DH, DH-D and DH-S ( $\mathrm{p}=0.002$, $\mathrm{p}=0.001, \mathrm{p}=0.002, \mathrm{p}=0.003$, respectively).

Blood serum glucose levels after hyperglycemia and anaesthesia were measured and similar results were found between study groups (Tab. 1)

\section{Discussion}

Emergency surgery rates in diabetic population during life span is reported as $5 \%$. Coronary artery bypass grafting, appendectomy, cholecystectomy, bariatric surgery, laparotomy, abscess drainage, embolectomy and lower extremity amputation are the most commonly performed procedures in this population. Although major procedures such as coronary artery bypass grafting have additional risks, minor interventions can be associated with unwanted surgical and anaesthetic outcomes (16).

Pre-anesthesic examination of the patients in diabetic population have to be done with extreme caution. In emergency situations, extensive medical history has to be learned and careful physical examination supported with blood studies have to be done. However, glycemic control in the diabetic patients at the time of emergency surgery is usually insufficient and suboptimal. So precautions have to be done, any impaired glucose level with blood electrolytes, signs of dehydration and acid-base derangements have to be identified before surgery. When identified once, insulin therapy with neutralization of dextrose solutions, correction of acid base disorders and electrolyte imbalances have to be treated (16).

Previous studies showed minimal increases in blood glucose levels with halothane in fed rats (17), and with isoflurane in fed dogs (18). However, in another study, authors showed significant increases in the blood glucose levels for a $3 \mathrm{hr}$ period in fed rats when compared to blood glucose levels determined in fasted rats ( $200 \mathrm{mg} / \mathrm{dl}$ versus $130 \mathrm{mg} / \mathrm{dl}$ ). These results are similar with those observed with ketamine-xylazine, although both studies have not investigated hyperglycemic states determined after isoflurane and ketamine-xylazine (19).

Acute hyperglycemia is an important prognostic factor in several critical illnesses. Many studies showed strong correlation with acute hyperglycemia and higher mortality rates in acute myocardial infarction $(20,21)$, cardiovascular surgery $(22)$ and stroke $(23,24)$. Similarly in animal models of myocardial infarction, deleterious effects of the high blood glucose levels were identified $(25,26)$. Saha et al (19) showed significantly elevated blood glucose levels in fed rats treated with ketamine plus xylazine (KX) and isoflurane. In contrast, they found that ketamine alone and pentobarbital sodium did not cause any increase in blood glucose levels in both fed and fasted rats. In KX and isoflurane group, decreased plasma levels of insulin, adrenocorticotropic hormone (ACTH), and corticosterone and increased levels of glucagon and growth hormone $(\mathrm{GH})$ were measured. When animals in the KX group were treated with yohimbin (specific alpha2 adrenergic receptor antagonist), they found significant alteration in serum levels of insulin, GH, ACTH and corticosterone. As a consequence, the authors concluded that hyperglycemic effect of KX was driven by its effect on specific alpha2 adrenergic receptors. Additionally, they recommended that agents such as the KX and isoflurane, which resulted in increased blood glucose levels in fed rats, had to be used with caution.

Carraretto et al (27) showed protective effects of propofol and isoflurane on ischaemia reperfusion injury while they couldn't show the same effect at transient hyperglycemic state. The transient hyperglycemia had a potential injury effect on the kidney after an episode of ischaemia reperfusion. The control of blood glucose levels may be clinically used for limiting organ dysfunction after periods of tissue ischaemia.

Efrati et al (28) studied halothane $2 \%$ and reported increased blood glucose levels between 5-60 minutes interval after induction and after $60^{\text {th }}$ minutes they showed decreasing glucose levels which returned pre-anaesthesia levels at 120th minutes.

In our study, sevoflurane and desflurane administrations at acute hyperglycemic state insignificantly increased blood glucose levels and these increased levels maintained during 24 hours postoperative period.

In conclusion, we can state that anaesthesia protocols in diabetics with acute hyperglycemia can increase the blood glucose levels - an insignificant, but numerically determined effect - and this effect may maintain longer than 24 hours and much longer follow up periods are needed in the patients with acute hyperglycemic state undergoing anaesthesia. Additionally we can conclude that both desflurane and sevoflurane can be used safely during acute hyperglycemic state, because of their non-increasing blood glucose effects. Future investigations are needed to further identify the effects of different anaesthesia and surgical protocols in acute hyperglycemia.

\section{References}

1. Robertshaw HJ, Hall GM. Diabetes mellitus: anaesthetic management. Anaesthesia 2006; 61: 1187-1190.

2. McAnulty GR, Robertshaw HJ, Hall GM. Anaesthetic management of patients with diabetes mellitus. Br J Anesth 2000; 85: 80-90.

3. McAnulty GR, Hall GM. Anaesthesia for the diabetic patient. Br J Anesth 2003; 88: 428-430.

4. Gu W, Pagel PS, Warltier DC, Kersten JR. Modifying cardio vascular risks in diabetes mellitus. Anesthesiology 2003; 98: 774-779.

5. Kadoi Y. Anesthetic considerations in diabetic patients. Part I: preoperative considerations of patients with diabetes mellitus. J Anesth 2010; 24: 739-747.

6. Marhoffer W, Stein M, Maeser D et al. Impairment of PMN leukocyte function and metabolic control of diabetes. Diabetes Care 1992; 15 : 256-260.

7. Lattermann R, Carli F, Wykes L, Schricker T. Perioperative glucose infusion and the catabolic response to surgery: the effect of epidural block. Anesth Analg 2003; 96: 555-562.

8. Schricker T, Gougeon R, Eberhart L et al. Type 2 diabetes mellitus and the catabolic response to surgery. Anesthesiology 2005; 102: 320-326.

9. Kennedy DJ, Butterworth JF 4th. Clinical review 57: Endocrine function during and after cardiopulmonary bypass: recent observations. J Clin Endocrinol Metab 1994; 78: 997-1002. 


\section{1-354}

10. Khan NA, Ghali WA, Cagliero E et al. Perioperative management of blood glucose in adults with diabetes mellitus. http://www.uptodate. com/contents/perioperative-management-of-blood-glucose-in-adults-withdiabetes-mellitus.

11. Desborough JP, Jones PM, Persaud SJ, Landon MJ, Howell SL. Isoflurane inhibits insulin secretion from isolated rat pancreatic islets of Langerhans. Br J Anesth 1993; 71: 873-876.

12. Gingerich R, Wright PH, Paradise RR. Inhibition by halothane of glucose-stimulated insulin secretion in isolated pieces of rat pancreas. Anesthesiology 1974; 40: 449-452.

13. Diltoer M, Camu F. Glucose homeostasis and insulin secretion during isoflurane anesthesia. Anesthesiology 1988; 68: 880-886.

14. David JS, Tavernier B, Amour J, Vivien B, Coriat P, Riou B. Myocardial effects of halothane and sevoflurane in diabetic rats. Anesthesiology 2004; 100: 1179-1187.

15. Kadoi Y. Blood glucose control in the perioperative period. Minerva Anestesiol 2012; 78: 574-595.

16. Dagogo-Jack S, Alberti GMM. Management of Diabetes Mellitus in Surgical Patients. Diabetes Spectrum 2002; 15: 44-48.

17. Heath DF, Frayn KN, Rose JG. Glucose turnover in the post-absorptive rat and the effects of halothane anesthesia. Biochem J 1977; 162: 653-657.

18. Horber FF, Krayer S, Rehder K, Haymond MW. Anesthesia with halothane and nitrous oxide alters protein and amino acid metabolism in dogs. Anesthesiology 1988; 69: 319-326.

19. Saha JK, Xia J, Grondin JM et al. Acute Hyperglycemia Induced by Ketamine/Xylazine Anesthesia in Rats: Mechanisms and Implications for Preclinical Models. Exp Biol Med 2005; 230: 777-784.
20. Capes SE, Hunt D, Malmberg K, Gerstein HC. Stress hyperglycemia and increased risk after myocardial infarction in patients without diabetes: a systematic overview. Lancet 2000; 355: 773-778.

21. Davies MJ, Lawrence IG. DIGAMI (diabetes mellitus, insulin glucose infusion in acute myocardial infarction): theory and practice. Diabetes Obes Metab 2001; 4: 289-295.

22. Van Den Berghe $\mathbf{G}$, Wouters $\mathbf{P}$, Weekers $\mathbf{F}$ et al. Intensive insulin therapy in critically ill patients. N Engl J Med 20001; 345: 1359-1367.

23. Scott JF, Robinson GM, French JM et al. Blood pressure response to glucose potassium insulin therapy in patients with acute stroke with mild to moderate hyperglycemia. J Neurol Neurosurg Psychiat 2001; 70: 401-404.

24. Bruno A, Williams LS, Kent TA. How important is hyperglycemia during acute brain infarction? Neurologist 2004; 10: 195-200.

25. Marfella R, D’amico M, Di Filippo C et al. Myocardial infarction in diabetic rats: role of hyperglycemia on infarct size and early expression of hypoxiainducible factor. Diabetologia 2002; 45: 1172-1181.

26. Shiomi T, Tsutsui H, Ikeuchi $M$ et al. Streptozotocin-induced hyperglycemia exacerbates left ventricular remodeling and failure after experimental myocardial infarction. J Am Coll Cardiol 2003; 42: 165-172.

27. Carraretto AR, Vianna Filho PT, Castiglia YM et al. Do propofol and isoflurane protect the kidney against ischemia/reperfusion injury during transient hyperglycemia? Acta Cir Bras 2013; 28 (3): 161-166.

28. Efrati S, Berman S, Hamad RA et al. Hyperglycaemia emerging during general anaesthesia induces rat acute kidney injury via impaired microcirculation, augmented apoptosis and inhibited cell proliferation. Nephrology 2012; 17: 111-122. 